Check for updates

Cite this: RSC Adv., 2019, 9, 20192

Received 25th April 2019

Accepted 11th June 2019

DOI: 10.1039/c9ra03102b

rsc.li/rsc-advances

\section{Preparation of a novel curcumin nanoemulsion by ultrasonication and its comparative effects in wound healing and the treatment of inflammation}

\author{
Niyaz Ahmad, (DD *ab Rizwan Ahmad, ${ }^{c}$ Ali Al-Qudaihi, ${ }^{\text {a }}$ Salman Edrees Alaseel, ${ }^{a}$ \\ Ibrahim Zuhair Fita, ${ }^{a}$ Mohammed Saifuddin Khalid ${ }^{d}$ and Faheem Hyder Pottoo ${ }^{d}$
}

\begin{abstract}
The aim of this study was to develop and evaluate a curcumin (Cur) nanoemulsion (NE) and enhance transdermal drug delivery. The comparative effects of Cur-NE were evaluated in terms of wound healing and anti-inflammatory action. Clove oil (oil), Tween-80 (surfactant), and PEG-400 (co-surfactant) were selected on the basis of their solubility and maximum nanoemulsion region. An aqueous micro-titration method with high-energy ultrasonication was used for the preparation of Cur-NE. This method was optimized to find the best NE, followed by a five-factor, three-level, central composite design. \% oil, \% $S_{\text {mix, }}$ ultrasonication time (min), ultrasonication intensity (\%), and temperature $\left({ }^{\circ} \mathrm{C}\right.$ ) were selected and optimized as independent variables. The optimized NE had parameters of $5.0 \%$ oil, $10 \% S_{\text {mix }}$, ultrasonication time (10 min), 40\% ultrasonication intensity and $50{ }^{\circ} \mathrm{C}$ temperature, which were applied as independent and dependent variables. On the basis of experimental data of the dependent variables, we calculated a hydrodynamic diameter of $93.64 \pm 6.48 \mathrm{~nm}$, transmittance of $98.64 \pm 0.37 \%$, and PDI of $0.263 \pm 0.021$. TEM and SEM results revealed the smooth and spherical shape of the particles in the NE, with a zeta potential of $-11.67 \pm 0.11$, refractive index of $1.71 \pm 0.034$, viscosity of $37 \pm 7 \mathrm{cp}, \mathrm{pH}$ of $7.4 \pm$ 0.07 , and drug content of $98.11 \pm 0.16 \%$ for the optimized Cur-NE. Cur-NE optimization with clove oil, Tween-80, and PEG-400 might be useful for enhancing the skin permeation of Cur. In conclusion, CurNE played a significant role in wound healing and exhibited anti-inflammatory effects, demonstrating its potential as a nanoformulation for safe and nontoxic transdermal delivery.
\end{abstract}

\section{Introduction}

Skin that has been physically injured, causing it to break or open, is known as a wound..$^{1,2}$ Repairing wounds is one of the most complicated physiological processes. Different types of cells contribute to wound healing (tightly regulated over time). The breakdown of this complicated pathway might be responsible for the failure of wound healing, leading to non-healing wounds. Wound repair involves several different steps, including inflammation and the generation of fresh tissues after the transformation of freshly formed tissue. All of these stages are completed on

\footnotetext{
${ }^{a}$ Department of Pharmaceutics, College of Clinical Pharmacy, Imam Abdulrahman Bin Faisal University, P. O. Box 1982, Dammam, Kingdom of Saudi Arabia-31441. E-mail: nanhussain@iau.edu.sa; niyazpharma@gmail.com; Fax: +966 13333 0290; Tel: +966 13333 5541; +966 531203626

${ }^{b}$ Department of Pharmaceutical Chemistry, College of Clinical Pharmacy, Imam Abdulrahman Bin Faisal University, Dammam, Kingdom of Saudi Arabia

${ }^{c}$ Department of Natural Products and Alternative Medicine, College of Clinical Pharmacy, Imam Abdulrahman Bin Faisal University, Dammam, Kingdom of Saudi Arabia

${ }^{d}$ Department of Pharmacology, College of Clinical Pharmacy, Imam Abdulrahman Bin Faisal University, Dammam, Kingdom of Saudi Arabia
}

different timescales, from minutes (i.e. first clotting followed by coagulation) to numerous months or many years. ${ }^{3,4}$ Pressure ulcers, arterial insufficiency, diabetic foot ulcers, and venous leg ulcers are all causes of chronic wounds. Chronic wounds can cause extreme morbidity and also mortality in older people that are affected by vascular diseases and diabetes mellitus. In general, older people can be successfully treated with surgery when they have nonhealing chronic wounds. Chronic wounds seriously affect quality of life and their treatment requires a very high quality of care. ${ }^{5,6}$ However, wounds can be treated by different drugs through parenteral or oral routes, but such systemic drug administration can cause serious side effects and can be rendered ineffective through the initial deactivation of the drug.

Curcumin exhibits antioxidant activity, which can play a great role in the treatment of wounds. ${ }^{7,8}$ Curcumin (a natural product), isolated from the rhizomes of Curcuma longa, is used orally and topically in the treatment of wounds. Curcumin (o-methoxyphenol derivative compound) possesses antioxidant activity and excites detoxification enzymes. Curcumin improves wound healing significantly when applied topically and also prevents oxidative damage. ${ }^{9}$ 
Curcumin enhances the production of granulation in body tissue with a greater amount of cellular content, promotes new vascularization and also increases the process of reepithelialization of wounds. ${ }^{10}$ Curcumin is poorly water soluble, meaning that it is restricted to the superficial stratum corneum (SC) after topical delivery. We can use the lipophilic materials (e.g. curcumin) that can increase the amount of drug at the required place and their direct delivery of curcumin into the skin avoiding any systemic side effects. Thus, a new formulation is required that releases curcumin in a sustained fashion for the effective and localized delivery of curcumin. Therefore, a formulation based on water that releases drug in a controlled manner, such as a curcumin-loaded nanoemulsion (Cur-NE), could be an effective approach for cutaneous wound healing. ${ }^{11}$ There have been so many investigations reported recently in which drugs are carried inside the core of NEs which easily penetrate into the skin via the subcutaneous barrier. ${ }^{12,13}$ Therefore, it is a novel approach to design a new formulation that can increase curcumin solubilization and increase its permeation via the skin. There are so many studies reported related to the improvement of solubility and dissolution with the use of different techniques, including polymeric nanoparticles, nanoemulsions, complexation, etc. ${ }^{13,14}$

Nanoemulsions (NEs) used as carrier systems for the encapsulation of active constituents are a novel drug delivery approach. Nanoemulsions are systems that are thermodynamically stable, and have a defined combination of surfactant/cosurfactant, oils, and water. ${ }^{13,15}$ When lipophilic drugs are applied topically, they are retained in the superficial skin layers. Local or systemic delivery of an active ingredient enhances its content by topical delivery of NEs using different types of mechanisms. ${ }^{8,16}$ NEs allow the entrapment of a higher quantity of drug in comparison to conventional topical preparations (e.g. creams, ointments, lotions, and gels). The solubility of poorly water-soluble drugs can be increased by NEs, in which drugs are dispersed in an oil droplet phase. Different compositions of the NE enhance penetration of drug through the diffusional barrier of the skin. ${ }^{17}$ The thermodynamic activity of the drug improves, which also helps in partitioning the skin. ${ }^{8}$ Therefore, a nanoemulsion can allow a drug to be transported beneath the skin layers of the wound, to the stratum germinativum and dermis, where angiogenesis occurs during wound healing. NEs can be used as a microreservoir in which the entrapped drug is released slowly.

Microemulsion and nanoemulsion were previously considered to be two interchangeable terminologies; now, the advancement in research has given new definitions to these terminologies. The use of more than $20 \%$ surfactant will give a microemulsion, which is more stable thermodynamically ${ }^{19}$ Moreover, microemulsions contain a very small globule size. ${ }^{20}$ However, a maximum concentration of the surfactant in a microemulsion fixes the limits that can be used for internal purposes. On the other hand, a nanoemulsion containing a very low concentration of surfactant is more appropriate for use with an internally administered drug. It was reported that $\mathrm{NE}$ is only stable kinetically. Now, most researchers report the need for external energy during the preparation of $\mathrm{NE}$, as NE-optimization is a nonspontaneous $(\Delta G>0)$ procedure. $^{21}$

This research offers a new chemical engineering concept, involving the use of oils and surfactant with ultrasonicationbased tailoring of nano-globule size, in order to achieve a curcumin nanoemulsion. Independent variables were optimized to produce the best curcumin-NE on the basis of globule size, PDI, and \% transmittance followed by the employment of a central composite design. The wound excision rat model is the best animal model for assessing wound healing effects, and this model is frequently used for essential oils. ${ }^{22}$ Essential oils can help invigorate your senses, and make you feel revitalized and energetic, leading to greater productivity. A few drops of potent essential oil, obtained from the flowers, leaves, seeds and bark of medicinal plants using steam distillation or cold pressing, can jolt you back on your feet when you are feeling depleted. There are thousands of research studies vouching for the remarkable healing and therapeutic benefits of essential oils. The aromatic molecular compounds in essential oils can pass right through the blood/brain barrier, directly affecting the areas in our brain that control feelings of stress, anxiety and mental fatigue. Aromatherapy is the best example of an alternative treatment system to improve the physical and psychological conditions of stressed individuals. ${ }^{46-48}$

In the proposed research study, the wound healing effects of an optimized curcumin-nanoemulsion (Cur-NE) were compared with those of pure Cur-S (reference), clove oil (reference) and a marketed preparation of antibiotic fusidic acid (brand name: Fusidin), using a wound excision rat model. Moreover, the optimized Cur-NE was used to further evaluate the inflammatory effects using a skin drug delivery system with an ex vivo skin permeation study. Cur-NE was developed and optimized with the use of safe and nontoxic ingredients such as clove oil, PEG-400, Tween-80, and water.

\section{Materials and methods}

\section{Materials}

Curcumin was obtained from RxBiosciences (18 908 Bonanza way, Gaithersburg, MD20879, USA). Milli-Q water was purified using a Milli-Q water purification system (ELGA, made in UK). PEG, Tween-80, and other surfactants were purchased from Sigma Life Science, Sigma Aldrich (Belgium). Methanol, ethanol, and acetonitrile (HPLCgrade; $99.9 \%$ purity) were procured from Sigma-Aldrich (Steinheim, Germany).

\section{Excipient screening for the preparation of final optimized nanoemulsion}

Oil, surfactant and co-surfactant were used as excipients and their selection was based on the solubility of curcumin, leading 
to a stable NE. Oleic acid, olive oil, almond oil, castor oil, clove oil, coconut oil, ethyl oleate, isostearyl isostearate and arachis oil were selected as oils. Based on biodegradability and easy accessibility, a very small number of oils was selected.

Tween-20, Labrasol®, Tween-80, Labrafil, PEG-400, PEG200, propylene glycol, carbitol and ethanol were selected as surfactants. Concisely, to $1.0 \mathrm{ml}$ oil or surfactant in microcentrifuge tube was added a maximum amount of Cur. After that, the tube was vortexed $\left(72.0\right.$ hour, $\left.25.0 \pm 1.0^{\circ} \mathrm{C}\right)$ using a Remi CM-101 cyclomixer. A maximum amount of Cur was removed by centrifugation (Kubota Laboratory Centrifuge) of the mixtures for (10.0 minutes $1008 \mathrm{rcf}$ ) followed by 72.0 hours. 10.0 microliters of supernatant were transferred to fresh tube and the volume was made up to $1.0 \mathrm{ml}$ with ethanol. The mixture was filtered first with the help of a syringe filter $(0.22 \mu \mathrm{m})$ followed by vortexing. We prepared a correct dilution for the UV-absorbance at $427 \mathrm{~nm}$ to plot a calibration curve, followed by an unidentified amount of Cur dissolved in a definite amount of oil or surfactant. ${ }^{23}$ We performed the excipient solubility study after that stability study of optimized Cur-NE.

\section{Pseudo-ternary phase diagram of preparation and optimization of placebo nanoemulsion}

Placebo nanoemulsion was prepared using an aqueous microtitration method. In brief, into a screw capped glass vial $(5.0 \mathrm{ml})$ was added the selected oil, surfactant, and cosurfactant $\left(S_{\text {mix }}\right)$. Oil and $S_{\text {mix }}$ were correctly mixed. The mixture was then titrated with Ultrapure Milli-Q Water so as to optimize the $\mathrm{NE}^{24}$ Various ratios of $S_{\text {mix }}(4: 1,1: 3,1: 1$, $1: 2,2: 1,5: 1$ and $3: 1$ ) were optimized for the amount of surfactant. Similarly, the amount of oil and $S_{\text {mix }}$ was also varied $(1: 9,1: 7,1: 8,1: 6,1: 3.5,1: 5,1: 3,2: 8,1: 2$, $4: 6,3: 7,6: 4,5: 5,8: 2,9: 1$ and $7: 3$ ) in the higher broader nanoemulsion region. On the basis of these permutations, every $S_{\text {mix }}$ ratio gave us 50 formulations, but finally, we prepared 99 total formulations based on 6 ratios of $S_{\text {mix }}$. As per the pseudo-ternary phase diagram region, all the formulations were optimized on the basis of the visual clarity of the microtitration and placebo nanoemulsions were prepared.

\section{Preparation and optimization of curcumin-loaded- nanoemulsion (Cur-NE)}

A high-energy ultrasonication technique was used to prepare the Cur-NE. Cur was dissolved in oil and $S_{\text {mix }}$ in a glass vial and a mixture was obtained which is microtitrated with Milli-Q-water to achieve a coarse emulsion. A $20 \mathrm{kHz}$ ultrasonic processor (Fisher Scientific Technology, USA) was used for the production of the supported nanoemulsion. A $20 \mathrm{kHz}$ ultrasonic processor consumes $500 \mathrm{~W}$ of power. This ultrasonic processor was prepared with a titanium horn (1.0 millimeter diameter) for the development of NE. In brief, Cur-NE was prepared with optimized parameters, i.e. ultrasonication intensity of $40.0 \%$, ultrasonication time of $10.0 \mathrm{~min}$, and temperature of $50{ }^{\circ} \mathrm{C}$, which were also used for a coarse Cur-emulsion. ${ }^{25}$ "Cavitation" is a phenomenon of driving force which can produce heat. ${ }^{26}$ Therefore, the selection of temperature $\left({ }^{\circ} \mathrm{C}\right)$ with time (min) is an important parameter for the preparation and optimization of Cur-NE. ${ }^{27,28}$ Cur-NE has a significantly smaller globule size $(r<100 \mathrm{~nm})$, with Brownian motion enhancement. Therefore, Cur-NE is kinetically stable, which is a requirement for the long-term storage of Cur-NE. ${ }^{20}$

\section{Experimental design used in Cur-NE}

Design Expert ${ }^{\circledR}$ (version 10.0.4, Stat-Ease, Minneapolis, USA) and its methodology surface response was used to optimize Cur-NE. CCD (central composite design) and BBD (Box-Benhnken design) are very important design experts used to optimize the parameters of nanoformulations in pharmaceutical research. CCD was chosen as the best one when we compared it to $\mathrm{BBD}$, in terms of response prediction. ${ }^{29} \mathrm{BBD}$ has some limitations in terms of recommendation, i.e., only low, middle and high values of independent variable formulation. However, CCD gives two extra values $(+\alpha \&-\alpha)$ which is a favorable design for rotatability requirements, including $\mathrm{BBD}$ values (Table 2$).^{30,31}$

For this research study, we selected the CCD tool for an optimization of nanoformulation. By the design expert (CCD), we optimized the globule size ( $\mathrm{nm}$ ), \% transmittance, and polydispersity index (PDI) as dependent variables, whereas oil (\%), $S_{\text {mix }}(\%)$, ultrasonication time (min), ultrasonication intensity $(\%)$, and temperature $\left({ }^{\circ} \mathrm{C}\right)$ were independent variables (IV). Moreover, \% oil and \% $S_{\text {mix }}$ were allocated as composition variables, whereas ultrasonication time, ultrasonication intensity, and temperature were taken as process variables. Composition of oil and surfactant were selected as high and low levels for the ternary phase diagram, but $-\alpha$ and $+\alpha$ levels were given by auto-selection of software. A total of 50 randomized formulations were run, as recommended by CCD, to feed values of IV. 32 were selected as factorial points, 8 were selected as axial points, and 10 were selected as center points out of the 50 recommended formulation runs (Table 2). Therefore, all the constraints correlate as per requirements for the optimization of the best formulation. CCD software generated

Table 1 Effect of selected oils and co-surfactants on zeta potential

\begin{tabular}{lllll}
\hline & & & \multicolumn{2}{c}{ Formulation } \\
Oil & Surfactant & Co-surfactant & code & Zeta potential \\
\hline \multirow{2}{*}{ Clove oil } & \multirow{2}{*}{ Tween 80} & PEG-400 & ClNE1 & $-11.67 \pm 1.021$ \\
& & PEG & ClNE2 & $-12.93 \pm 1.053$ \\
& & Carbitol & ClNE3 & $-13.21 \pm 1.038$ \\
& & Labrasol & ClNE4 & $-13.99 \pm 1.213$ \\
Coconut oil & Tween 80 & PEG-400 & CoNE1 & $-13.51 \pm 1.029$ \\
& & PEG & CoNE2 & $-13.81 \pm 1.091$ \\
& & Carbitol & CoNE3 & $-14.01 \pm 1.380$ \\
Olive oil & \multirow{2}{*}{ Tween 80 } & PEG-400 & OlNE1 & $-12.01 \pm 1.031$ \\
& & PEG & OlNE2 & $-13.09 \pm 1.078$ \\
& & Carbitol & OlNE3 & $-15.21 \pm 1.071$
\end{tabular}


Table 2 Variables in "Design Expert" software for preparation and optimization of curcumin-nanoemulsions (Cur-NE)

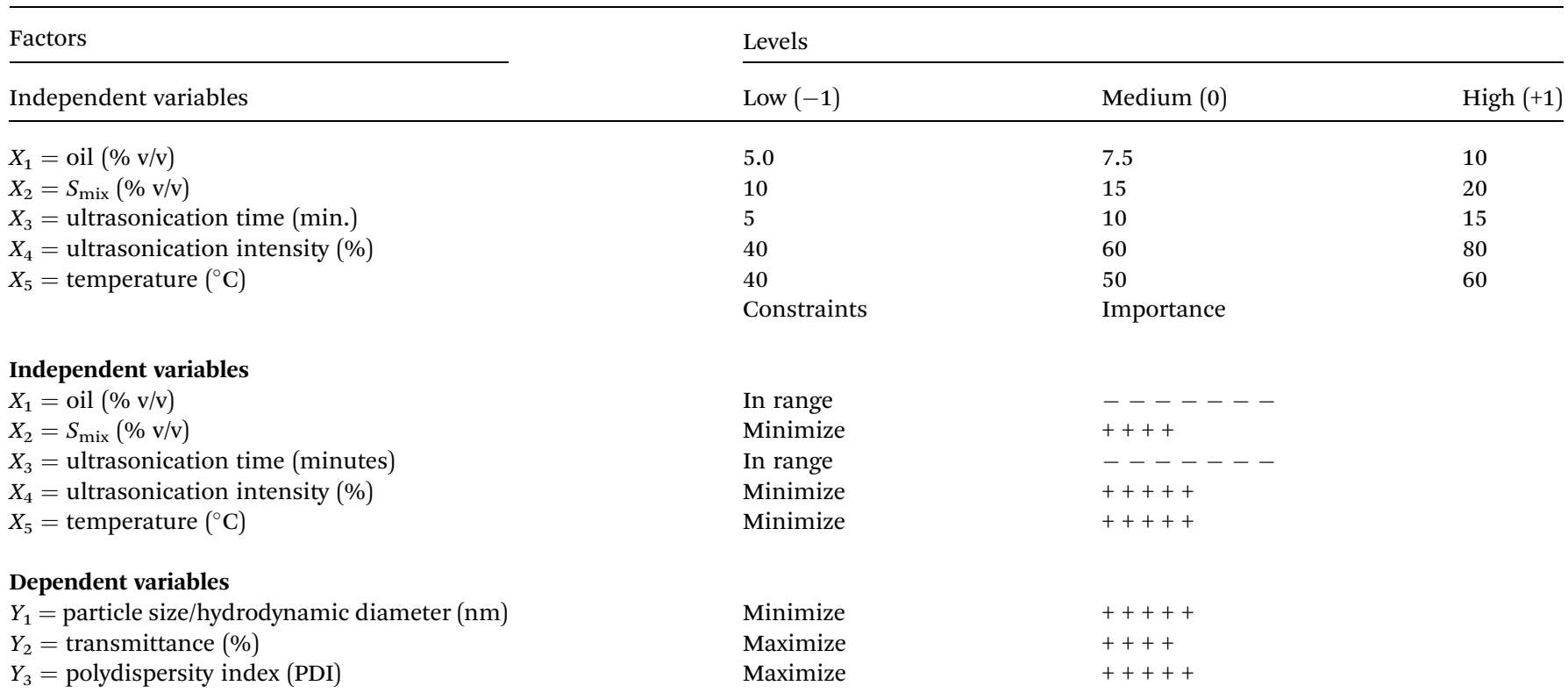

a quadratic polynomial equation for five-factor three-level, which is outlined below:

$$
\begin{gathered}
Y=b_{0}+b_{5} X_{5}+b_{6} X_{6}+b_{7} X_{7}+b_{56} X_{5} X_{6}+b_{57} X_{5} X_{7}+b_{57} X_{5} X_{7}+ \\
b_{55} X_{5}^{2}+b_{66} X_{6}^{2}+b_{77} X_{7}^{2} .
\end{gathered}
$$

\section{Optimized Cur-NE characterization by the use of dyes}

A classic dye test was performed to confirm the preparation of Cur-NE. A water soluble dye, methylene blue, and water insoluble methyl red, were used as dyes. $2.0 \mathrm{mg} \mathrm{mL}^{-1}$ of ethanolic solution was also prepared..$^{32}$ All the dye solutions $(100.0$ microliter) were transferred to borosilicate glass containing nanoemulsions and the nanoemulsions were visually observed for 1 hour. $^{32}$

\section{Nanoemulsion characterization}

Droplet size (DS) and PDI measurements. A Malvern Zetasizer Nano ZS (Malvern Instruments, Malvern, UK), utilizing DLS (dynamic light scattering) at $25^{\circ} \mathrm{C}$, was used to measure the

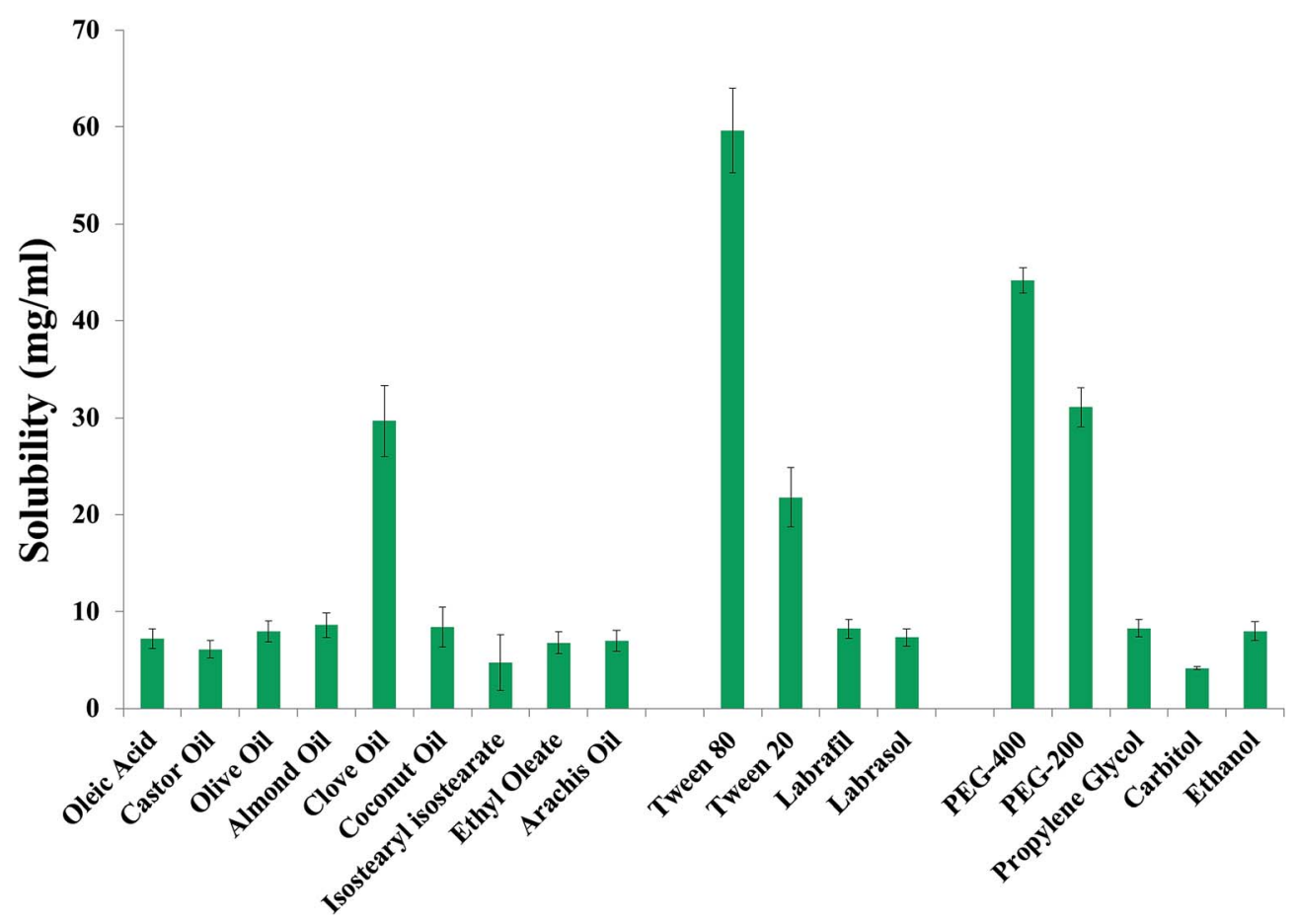

Fig. 1 Percentage nanoemulsion region obtained for different surfactants and co-surfactants. 


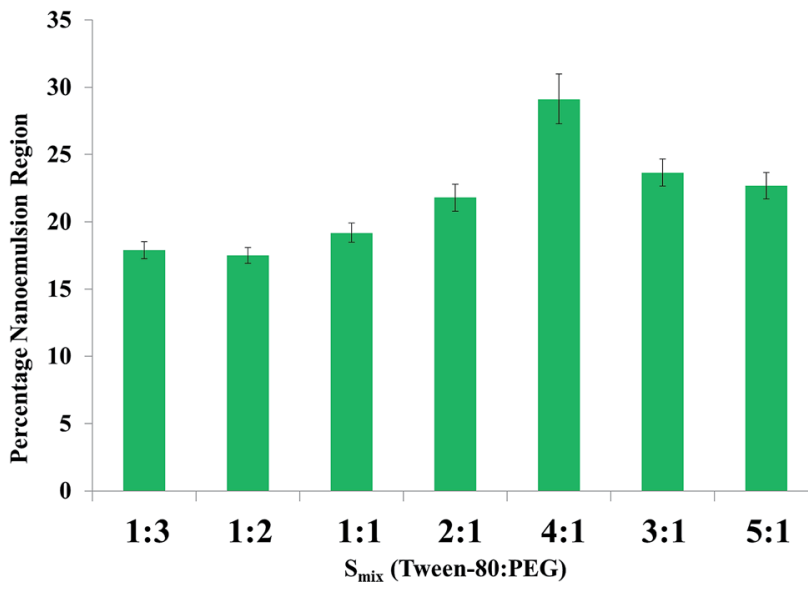

Fig. 2 Percentage nanoemulsion region obtained for different $S_{\text {mix }}$ ratios.

mean DS and PDI. A disposable capillary cuvette (DTS 1060) equipped with an electrode was used for the observation of particle size. As mentioned by Ahmad et al., before measurement the samples were diluted 100 -fold with double-distilled water in order to avoid multiple scattering effects. An average of 13 measurements at an angle of $173^{\circ}$ were taken to calculate the DS and PDI, as mentioned by Ahmad et al..$^{13,32-34,36}$

Particle surface charge (zeta potential) measurements. The Malvern Zetasizer Nano ZS (Malvern Instruments, Malvern, UK), maintained at 1.33 and $0.89 \mathrm{cp}$ for refractive index and viscosity respectively in order to mimic the value of pure water, was applied to measure the zeta potential through electrophoretic mobility measurements. Immediately after the DS measurements, the potential was measured using the same cuvette with three successive readings for each sample, and the mean value and standard were calculated as per Ahmad et al. . $^{13,32-34,36}$

Shape and surface morphological analysis by SEM and TEM. TEM (Morgagni 268D; FEI Company, Hillsboro, OR) was used to determine the surface morphology of prepared Cur-NE. Briefly, a drop of NE was placed on a paraffin sheet for sticking of Cur$\mathrm{NE}$ (1 min). It was then covered with a copper grid, followed by the placement of the grid (>5 s) in a drop of phosphotungstate.
Air drying of the sample was performed, while any traces of solution remaining were cleared using filter paper. The sample was observed again using TEM.

SEM (Zeiss EVO40; Carl Zeiss, Cambridge, UK) was used to determine the surface texture of optimized Cur-NE. Briefly, a double-sided conductive tape was used for sample spreading. Then, the surface was coated with gold using a SCD020 Blazers sputter coater unit (BAL-TEC GmbH, Witten, Germany) under high vacuum. The sputter unit was pre-maintained with Argon gas for $100 \mathrm{~s}$ at $50 \mathrm{~mA}$.

$\%$ transmittance. In order to get an idea regarding the globule size and stability of the formulation, \% transmittance $(650 \mathrm{~nm})$ for the developed formulations was observed spectrophotometrically (Shimadzu, UV-1601, Kyoto, Japan). The blank used was distilled water and no dilution for any formulation was carried out in this process. ${ }^{32,33}$

In vitro drug release. In vitro release of drug from optimized $\mathrm{NE}$ was performed using a pre-treated dialysis membrane (pore size: $2.4 \mathrm{~nm}$; molecular weight cut-off $\sim 12-14 \mathrm{kD}){ }^{34,35}$ First, the release medium was prepared (phosphate buffer; $\mathrm{pH} 7.4=$ $100.0 \mathrm{~mL}$ ). The temperature was maintained at $37.0 \pm 1.0{ }^{\circ} \mathrm{C}$ with the help of a stirrer for up to $6 \mathrm{~h}$ at $1 \mathrm{rcf}$. Finally, the dialysis bag was properly checked for any leaks and Cur-NE (containing $0.50 \mathrm{mg}$ Cur) was placed inside. For the Cur-release study from Cur-NE, time points were selected $(30,60,120,240,360,480$, $720,1440 \mathrm{~min}$ ). At every time point, we withdrew test samples (1 $\mathrm{mL})$. All the withdrawn samples were filtered using a syringe filter $(0.45 \mu \mathrm{m})$ first. After that, the Cur quantity was analyzed through the in-house reproduced UHPLC-method. ${ }^{36}$

Refractive index (RI) and viscosity. Refractive index (RI) and viscosity test was performed using the method of Ahmad et al. ${ }^{32,33}$

Differential scanning calorimetry (DSC). DSC 214 Polyma (NETZSCH-Wittelsbacherstraße 42, 95100 Selb, Germany) was used for entrapment studies, which were based on the thermal analysis of Cur, Tween-80, PEG-400, clove oil, and optimized Cur-NE. In brief, the samples were kept in the pan $(10.0 \mathrm{mg})$, whereas an empty pan was taken as a reference. For the instrument, we selected a temperature range $\left(40.0^{\circ} \mathrm{C}\right.$ to 400.0 ${ }^{\circ} \mathrm{C}$ ) that is based on a temperature increase as determined as 10 $\mathrm{K} \mathrm{min}^{-1}$ with the help of nitrogen flow (maintained 60.0

Table 3 Results of regression analysis for responses $Y_{1}$ (particle size, nm), $Y_{2}$ (transmittance, \%) and $Y_{3}$ (PDI)

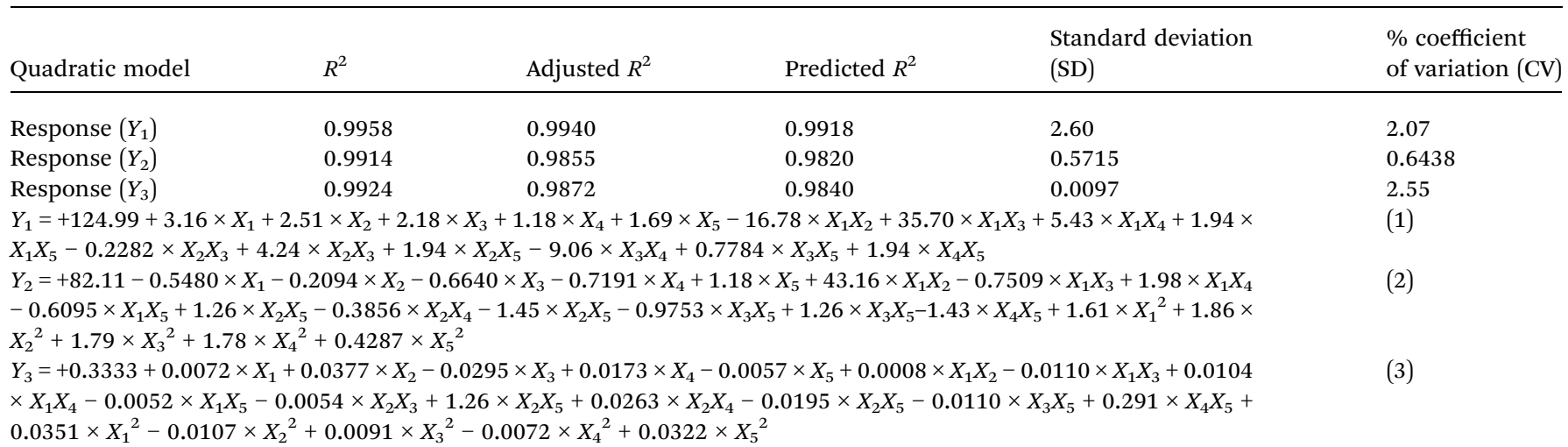


A
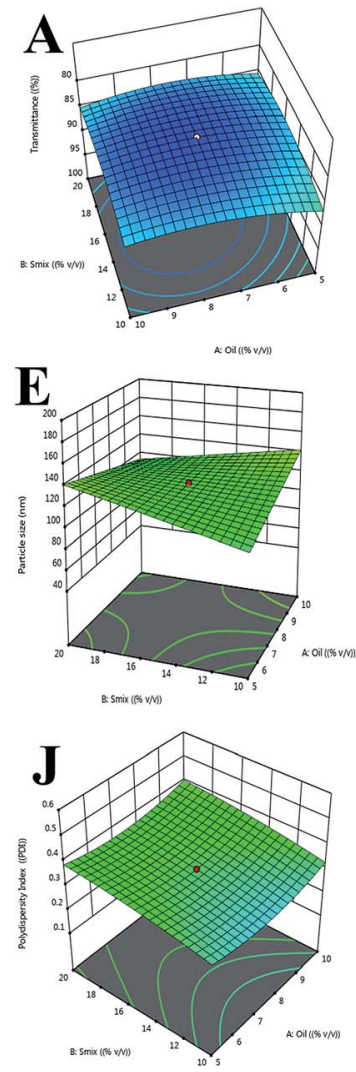

B

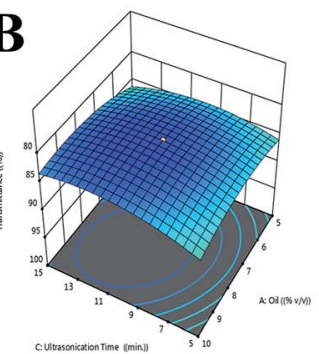

$\mathbf{F}$
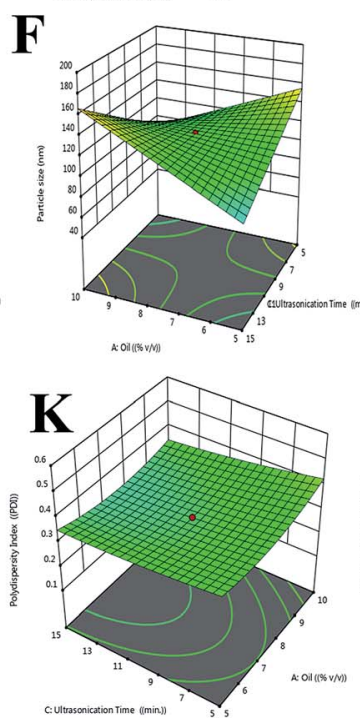
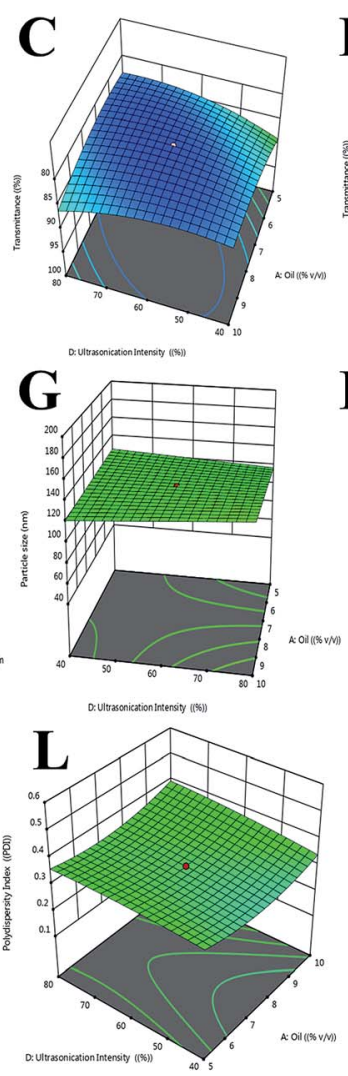

D

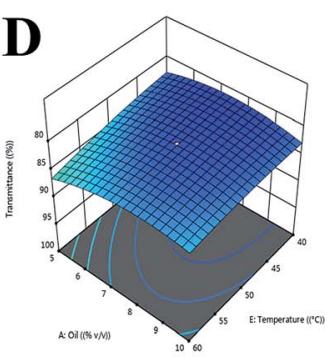

H
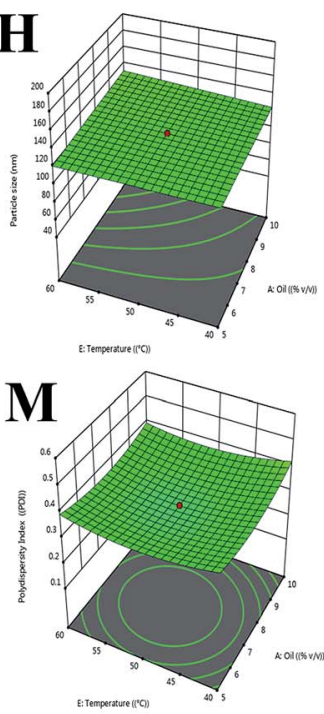
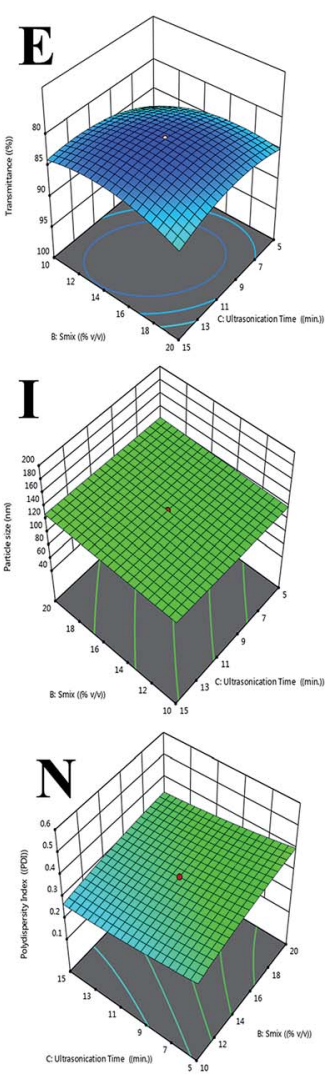

Fig. 35 -D response surface plots showing the interaction effect for particle size, \% transmittance and PDI as a function of oil (\%), $S_{\text {mix }}(\%)$, ultrasonication time (min), ultrasonication intensity $(\%)$, and temperature $\left({ }^{\circ} \mathrm{C}\right)$.

$\left.\mathrm{ml} \mathrm{min}^{-1}\right){ }^{37}$ DSC 214 Polyma (NETZSCH-PROTEUS-70, Germany) software was used to obtain the values.

Permeability skin study (ex vivo). We were followed the Ahmad et al. method to perform the skin permeation study. ${ }^{32,33}$

In vivo study. For the in vivo pharmacodynamics and histopathological studies, a proper ethical approval was obtained from the Animal Ethical Committee, Imam Abdulrahman Bin Faisal University (Dammam-Saudi Arabia). Albino rats (weight; 180-200 g) were grouped (5 to 10 in each cage) and maintained in a natural light and dark cycle with free access to food and water (temperature 20.0 to $30.0{ }^{\circ} \mathrm{C}$; humidity 50.0 to $55.0 \%$ ). All animals were kept under laboratory prescribed conditions. Research activity was started during the light cycle in the waking condition using freely moving animals.

Histomorphological evaluation of tissues. Healed tissue from each group of rats was taken at the end of the experiment (after 24 days) to perform the histomorphological analysis, using formalin $(10.0 \%)$, dehydrated alcohol with paraffin blocks. Xylene was used to deparaffinize the tissue sections. A microtome was used to cut sections of specific diameters, which were then stained by HE (Hematoxylin Eosin), followed by analysis using a light microscope. Inflammation was identified blindly in the healed areas, while infiltrations or their field of inflammatory cells were counted. The healed area of tissues was evaluated by inflammatory cell infiltration, epithelization, neovascularization, fibroblast proliferation, and collagen deposition. We used a modified version of a reported scale (0.05.0) to characterize the wound healing area. ${ }^{33,41}$

Evaluation of wound healing area. Evaluation of the wound healing area was performed in Group I (the control group: optimized nanoemulsion without Cur loaded), Group II (pure clove oil: $10 \mathrm{mg} \mathrm{kg}^{-1}$ body wt), Group II (pure Cur-S: $10 \mathrm{mg} \mathrm{kg}^{-1}$ body wt), Group III (optimized nanoemulsion; Cur-NE: $10 \mathrm{mg}$ $\mathrm{kg}^{-1}$ body wt) and a marketed preparation of the antibiotic Fusidic Acid (Fusidin: $10 \mathrm{mg} \mathrm{kg} \mathrm{kg}^{-1}$ body wt) serving as the positive control. Wistar rats (female albino; weight: 200.0-250.0 g) were used in this research. Five groups ( 6 rats in each group; 6 $\times 5=35$ ) were used in this study for the evaluation of the wound healing area. Before making the wounds on the rats, we performed anesthesia with epilation. Excision of the wound was performed, with an approximate predetermined area of 500 $\mathrm{mm}^{2}$ maximum thickness on the anterior-dorsal side of each female rat. ${ }^{3,38}$ All the female rats topically received the test formulations once daily for their respective group, for 24 days. The percentage of wound contractions and their healing time were the best parameters to analyze the wound healing. We determined the wound area on every third day by using a butter paper and drawing the wound area. The percentage area of wound contractions were calculated using a graph sheet. ${ }^{33,38}$ Total wound healing area and closing area were determined.

Cur-NE treatment for inflammation. All the rat groups were further divided into groups of two rats in each cage with weights 

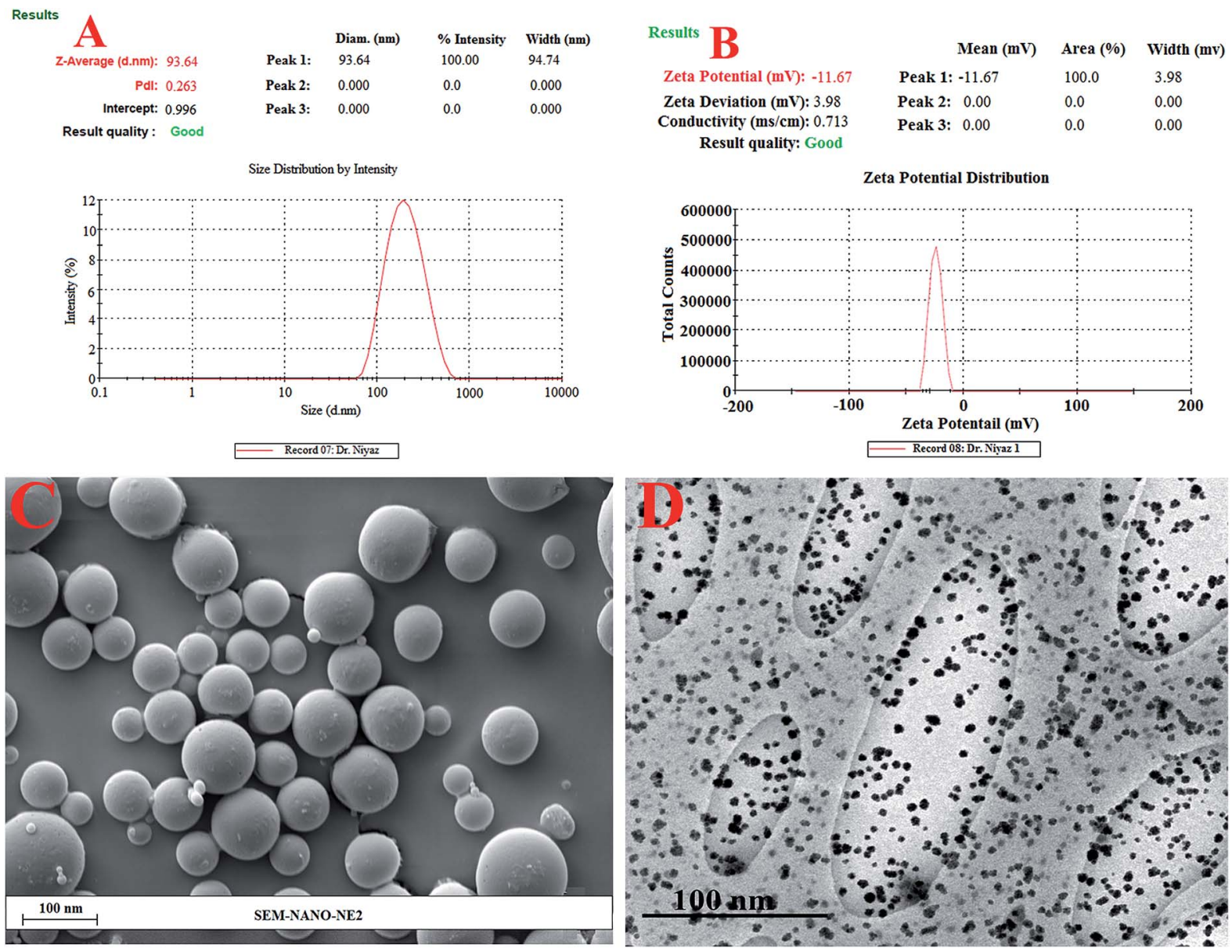

Fig. 4 Dynamic light scattering techniques for determining the particle size distribution of Cur-NE (A) and zeta potential of Cur-NE (B). Scanning Electron Microscopy (SEM) (C), and Transmission Electron Microscopy (TEM) (D) images.

of 200.0-250.0 g. At all times the rats had unlimited access to food and water, while the temperature was maintained at 20.0$30.0{ }^{\circ} \mathrm{C}$, the humidity was $50.0-55.0 \%$, and a natural light and dark cycle was employed. Finally, we maintained these laboratory conditions when performing the experiments during the waking cycle of the freely moving rats. For each group of rats where epilation was previously performed on the skin of the dorsal side, Cur-NE or Cur-S (one teaspoonful) was applied topically. Carrageenan injection (1\% normal saline suspension; 100.0 microliter) was applied beforehand to promote inflammation in the right hind paw. ${ }^{33,39}$

In the left hind paw, the same amount of non-pyrogenic normal saline solution was injected as a control. Paw thickness was evaluated every $4 \mathrm{~h}$ through Digital Plethysmograph (UGO Basile, Italy) and the percentage change of edema was also calculated. Each female rat served as its own control. Paw thickness (control means saline-injected) was evaluated for each measurement. This was compared with the inflamed paw for each female rat to evaluate all sampling time points.

Statistical analysis. All outcomes were evaluated and represented as mean \pm SEM (standard error of mean). Student's $t$-test was used for the unpaired observations calculation and the difference with the help of ANOVA, i.e. $p$-value.

\section{Results and discussion}

\section{Excipient screening for the preparation of final optimized nanoemulsion}

Curcumin solubility was determined in various oils (oleic acid, castor oil, olive oil, almond oil, clove oil, coconut oil, isostearyl

Table 4 Best optimized and predicted batch of Cur-NE with independent variables, and dependent variables

\begin{tabular}{|c|c|c|c|c|c|c|c|c|}
\hline \multirow[b]{2}{*}{ Batch } & \multicolumn{5}{|c|}{ Independent variables } & \multicolumn{3}{|c|}{ Dependent variables } \\
\hline & $X_{1}$ & $X_{2}$ & $X_{3}$ & $X_{3}$ & $X_{3}$ & $Y_{1}$ & $Y_{2}$ & $Y_{3}$ \\
\hline Optimized & 5.00 & 10 & 45 & 40 & 60 & $93.64 \pm 6.48$ & $98.64 \pm 0.37$ & $0.263 \pm 0.021$ \\
\hline
\end{tabular}


Table 5 Some other characterized parameters of CUR-NE

\begin{tabular}{llllll}
\hline PDI & Zeta potential $(\mathrm{mV})$ & Refractive index & Viscosity (centipoise) & pH & Drug content (\%) \\
\hline $0.263 \pm 0.021$ & $-11.67 \pm 0.11$ & $1.71 \pm 0.034$ & $37 \pm 7 \mathrm{cp}$ & $7.4 \pm 0.07$ & $98.11 \pm 0.16 \%$
\end{tabular}

isostearate, ethyl oleate and arachis oil) as displayed in Fig. 1. Finally, we selected four oils (clove oil, coconut oil, almond oil and olive oil) that showed the maximum solubility. As shown in Fig. 1, Tween-80 with the HLB value of 15.00 exhibited the maximum solubility, followed by PEG-400. Because of its molecular weight, Tween-80 has a reduced particle size when compared with other polymeric surfactants. ${ }^{40}$ On the basis of these properties, Tween-80 was selected as the surfactant.

The zeta potential is also an important parameter to determine in order to estimate the total surface charge and stability of the optimized Cur-NE. For the optimized Cur-NE formulation, the zeta potential values were found to be $-11.67 \pm 1.021$ to $-15.21 \mathrm{mV}$ (Table 1 ). The smallest zeta potential value was identified for the Cur-NE ClNE1, i.e. $-11.67 \pm 1.021 \mathrm{mV}$. The highest value of the zeta potential was found for the Cur-NE OlNE3 $(-15.21 \pm 1.071)$. The negative zeta potential values in each Cur-NE may be due to the presence of negatively charged fatty acid esters in clove oil. ${ }^{41}$ The zeta potential values of the optimized Cur-NE formulations observed experimentally in the magnitude of $\pm 15 \mathrm{mV}$ showed the Cur-NE stable formulation (Table 5). ${ }^{\mathbf{4 1}}$

Additionally, oil and co-surfactant screening was based on the zeta potential to prepare a stable nanoformulation. The CINE1 formulation showed the lowest zeta potential $(\zeta=$ $-11.67 \mathrm{mV}$ ) as presented in Table 1 , which is based on the surfactants Tween-80 and PEG-400 along with clove oil, followed by a final selection of combination. Clove oil was selected due to its renowned health benefits, i.e. wound healing, antiinflammatory, antioxidant, anticancer, and cerebral ischemia. ${ }^{\text {41,42 }}$ Furthermore, its nanoemulsions are minimally prone to Ostwald ripening, as mentioned by Alam et al., ${ }^{41}$ who observed that clove oil has more effective anti-inflammatory action in treating periodontitis. ${ }^{\mathbf{4 1 , 4 3}}$

Tween-80 and PEG-400 combination of surfactants and cosurfactants produced a very transparent NE. This combination was previously used by other studies ${ }^{32,34}$ in which a co-surfactant with a hydrophilic nature produced the transparent microemulsion. These microemulsions exhibited a very small zeta potential. Non-ionic surfactants will produce a very small zeta potential on the charge of the surface, followed by reducing the particle size of $\mathrm{NE}^{\mathbf{4 4}}$

\section{Pseudo-ternary phase diagram used for preparation and optimization of placebo nanoemulsion}

Various ratios of $S_{\text {mix }}$ were graphically represented on the basis of pseudo-ternary phase diagrams, i.e. the nanoemulsion region shown in Fig. 2 can be clearly seen. $S_{\text {mix }}$ ratios of $1: 2$ to $4: 1$ were achieved by increasing the concentration of surfactant, which led to an increase in the nanoemulsion region. $S_{\text {mix }} 1: 2$ exhibited the lowest nanoemulsion region, while $S_{\text {mix }} 4: 1$ showed the maximum nanoemulsion region. Expansion of the nanoemulsion region is due to parallel increment of the HLB scale value, i.e. 15 for Tween-80. The higher the HLB value of $S_{\text {mix }} 4: 1$, the greater the viscosity of the nanoformulation with the highest nanoemulsion region. ${ }^{34,37}$ Lowest and highest percentages of $S_{\text {mix }}$ and oil were determined by the pseudoternary phase diagram.

Numerous combinations of oil, water and $S_{\text {mix }}$ were scrutinized; however, the best two combinations were selected finally. Few of the combinations, especially those with a greater percentage of oil as compared to water, were discarded due to

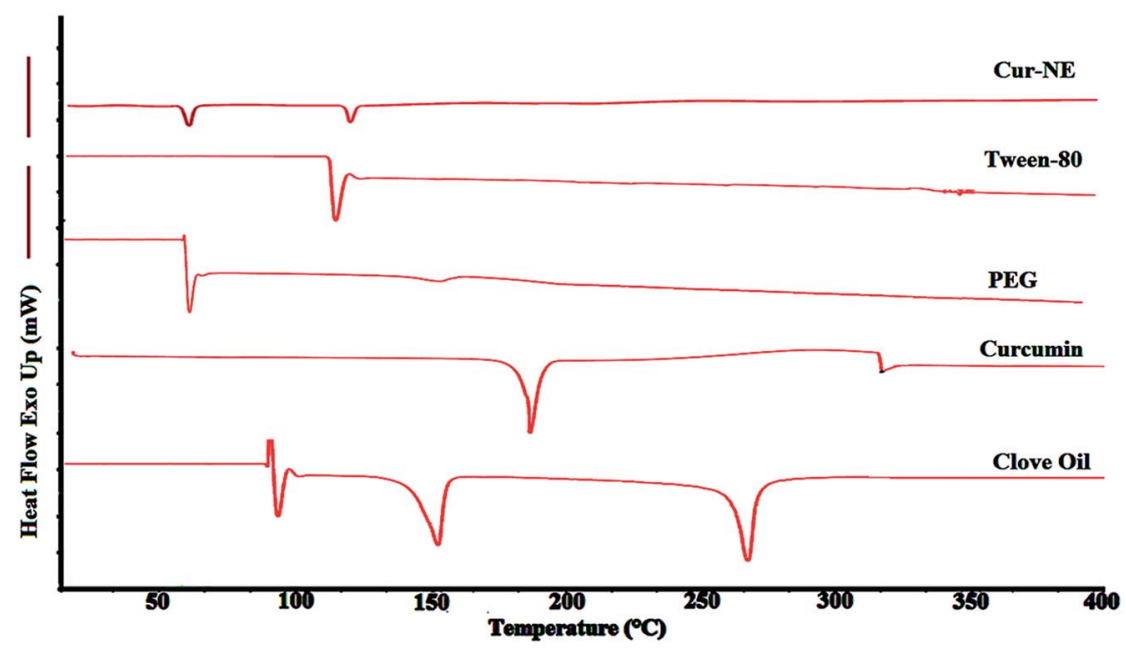

Fig. 5 Endothermic DSC thermograms of clove oil, pure curcumin, polyethylene glycol-400 (PEG-400), Tween-80, and lyophilized-optimizedCur-NE. 

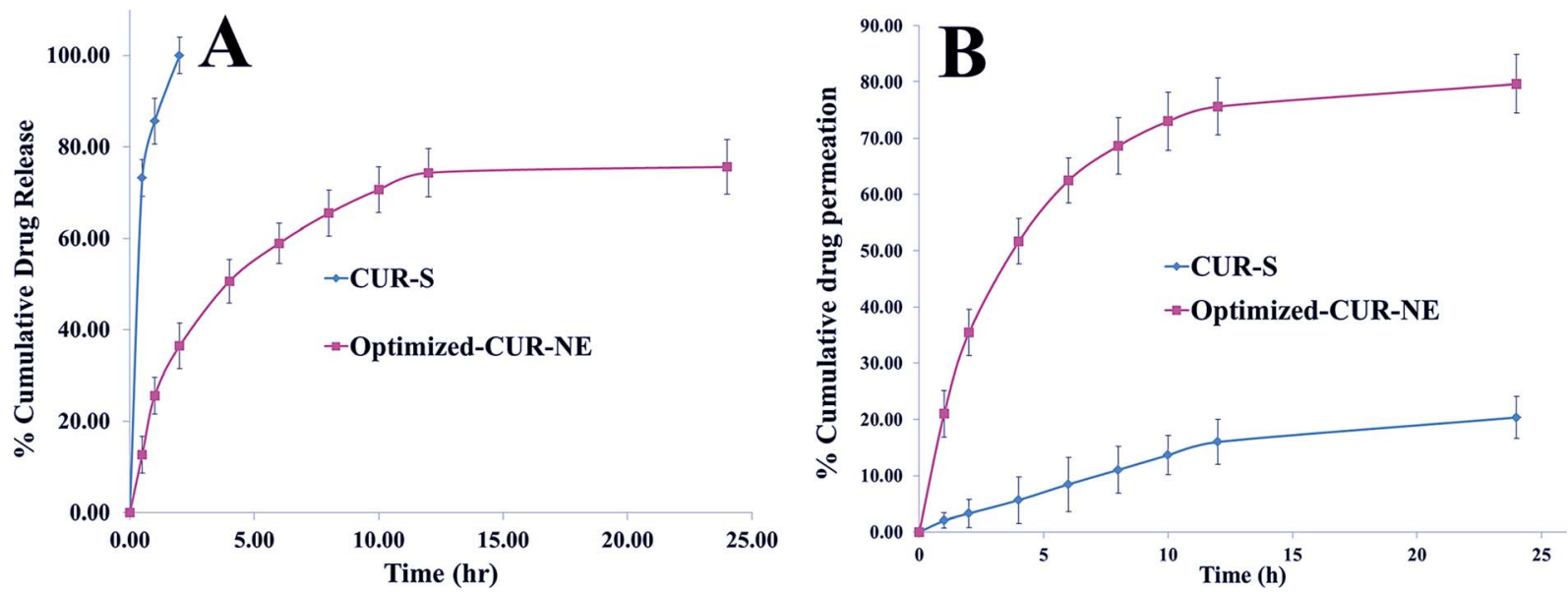

Fig. 6 (A) The cumulative percentage release of curcumin from Cur-NE as compared to pure curcumin. (B) In vitro skin permeation profiles of developed Cur-NE as compared to pure curcumin through rat skin.

the possibility of water in oil (w/o) emulsion formation. The minimum percentage of oil used was $5.0 \%$, as below this amount the drug dissolving potential of the oil was lost. Meanwhile, the maximum amount of oil used was $10.0 \%$, as above this concentration the surfactant percentage crosses the
$20 \%$ mark, thus favoring the formation of a microemulsion rather than a nanoemulsion, as reported by Tadros et al. ${ }^{19}$ The same concept is supported by McClement's statement, i.e. "to prepare a microemulsion a greater surfactant to oil ratio is required as compared to nanoemulsion". ${ }^{20,33,37}$

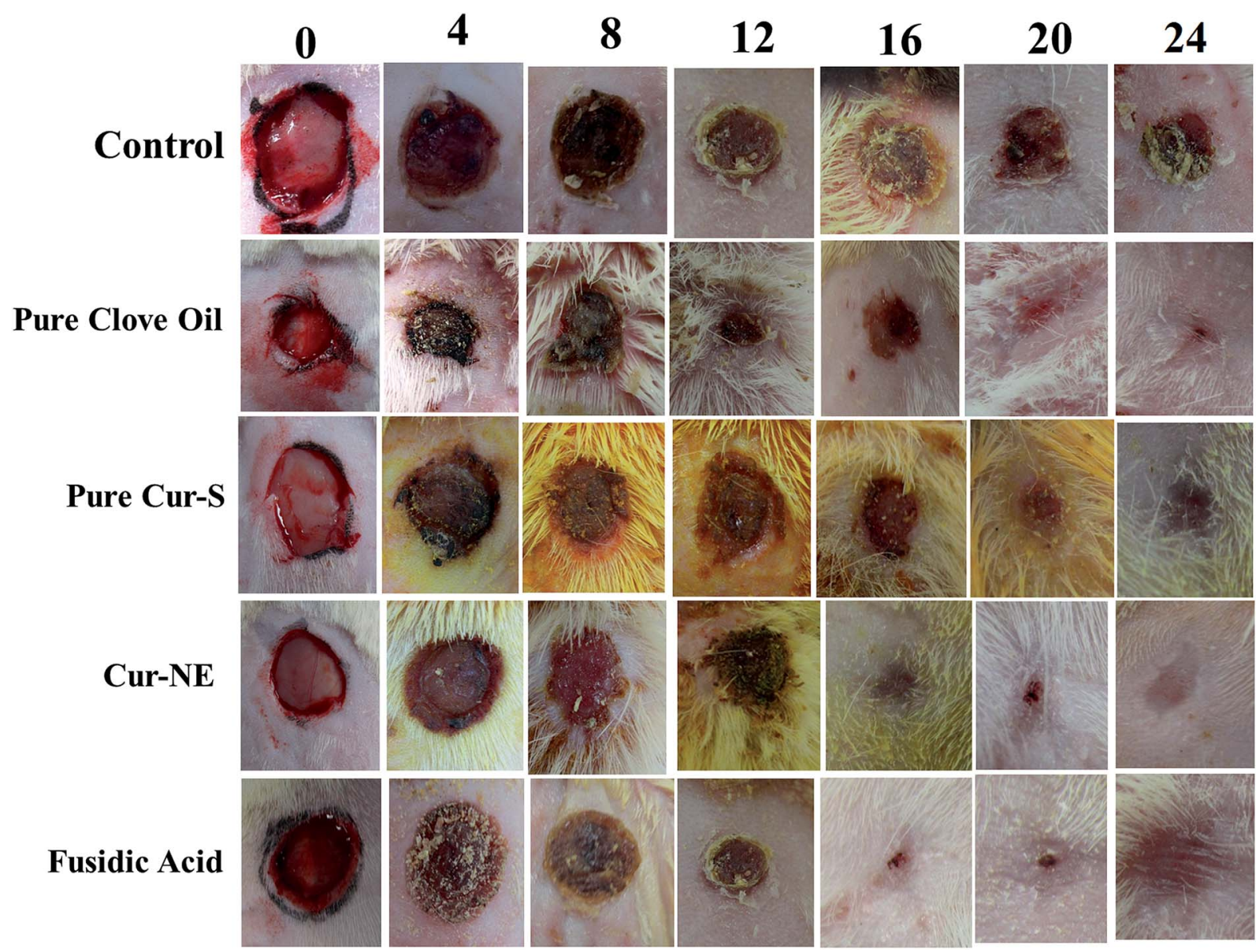

Fig. 7 Wound healing effects of optimized nanoemulsion without Cur loaded; pure clove oil; pure Cur-S; optimized nanoemulsion and marketed preparation of antibiotic fusidic acid (Fusidin; positive control) in comparison with the control after 0, 4, 8, 12, 16, 20 and 24 days of inducing wound healing. 


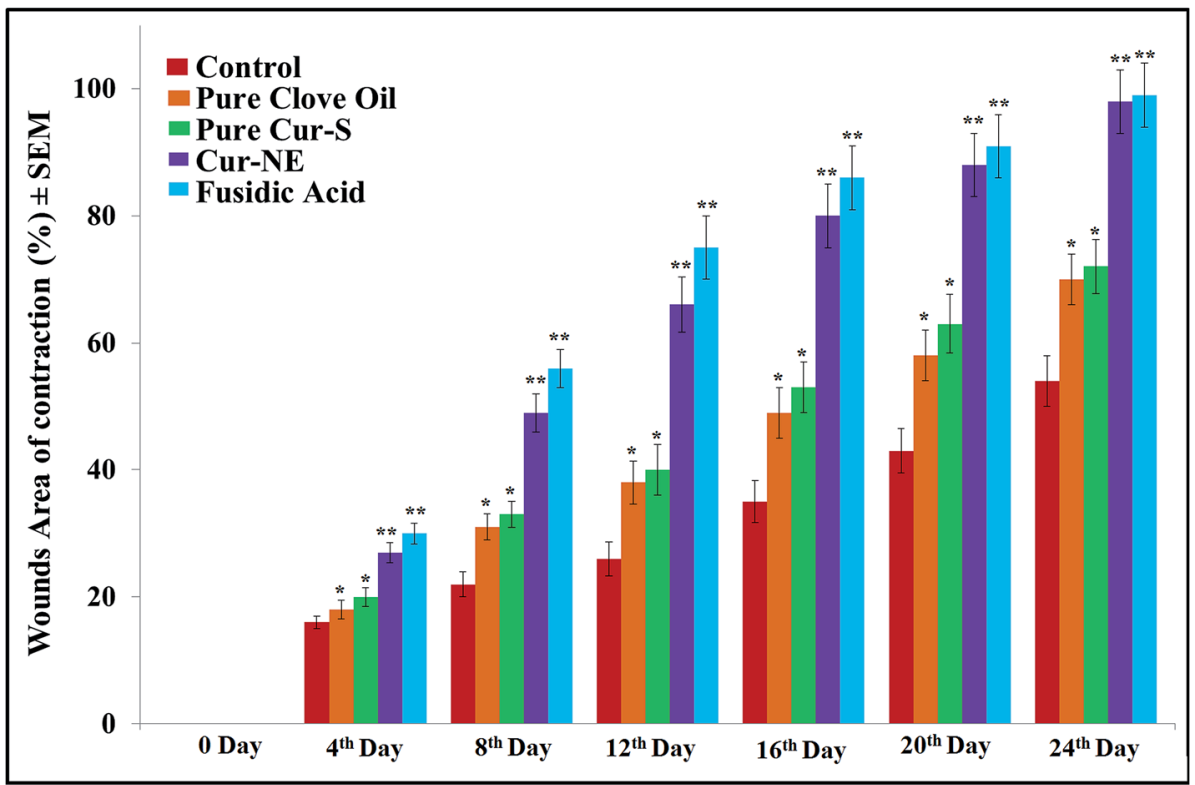

Fig. 8 Influence of oral administration of optimized nanoemulsion without Cur loaded; pure clove oil; pure Cur-S; optimized nanoemulsion and marketed preparation of antibiotic fusidic acid (Fusidin; positive control) on circular excision wound model in rats at different days of treatment. $* P<0.01$ compared to control.

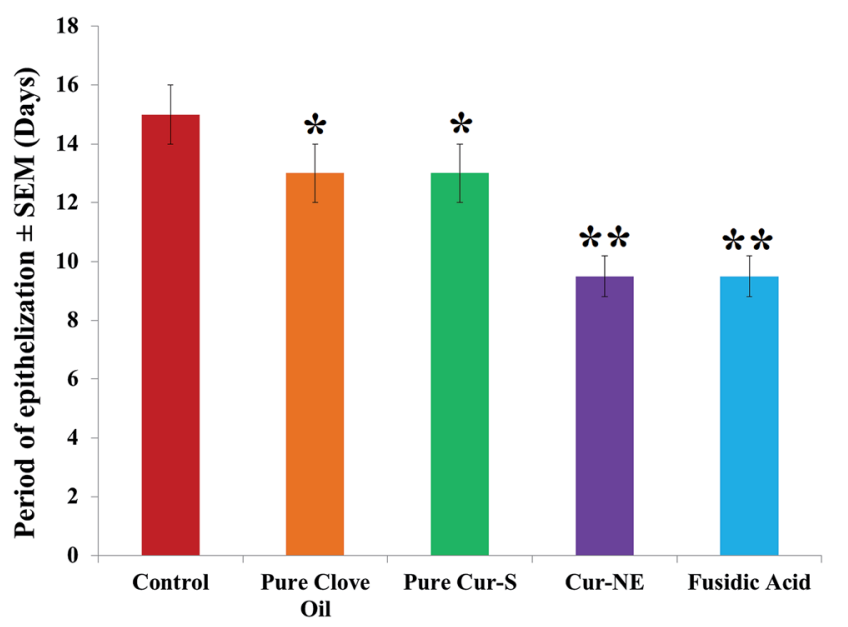

Fig. 9 Influence of oral administration of optimized nanoemulsion without Cur loaded; pure clove oil; pure Cur-S; optimized nanoemulsion and marketed preparation of antibiotic fusidic acid (Fusidin; positive control) on wound epithelization period. $* P<0.05$ compared to control.

Based on this supportive information, the lower and upper surfactant limit as selected was $5-10 \%$ respectively, which further strengthened the design for optimization. Ultrasonication was used to prepare Cur-NE and was also selected as the best method on the basis of previously reported literature. ${ }^{13}$ This method can be very clearly defined on the basis that ultrasonication did not interrupt the integrity of the nanoemulsion globules. In addition, for the nanoemulsion formula optimization, oil, $S_{\text {mix }}$, ultrasonication time (min), ultrasonication intensity (\%), and temperature $\left({ }^{\circ} \mathrm{C}\right)$ were chosen as five independent variables.

\section{Experimental design model: fitting and optimization of Cur- NE}

On the basis of the above-mentioned initially reported studies, the independent variables were determined, for example oil $(\%), S_{\operatorname{mix}}(\%)$, ultrasonication time (min), ultrasonication intensity (\%), and temperature $\left({ }^{\circ} \mathrm{C}\right)$. The experimentally observed values for globule size $\left(Y_{1}: 50.85-188.60 \mathrm{~nm}\right), \%$ transmittance $\left(Y_{2}: 81.76-98.64 \%\right)$ and PDI $\left(Y_{3}: 0.256-0.559 \%\right)$ were determined. All the obtained data were kept in the software to optimize all of the three dependent variables (DV), following polynomial quadratic optimization models $(p<$ 0.001). All the significant and non-significant variables are shown in Table 3, if there is any adjustment for all three DV with

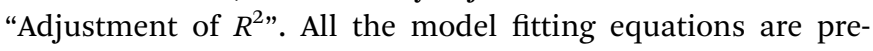
sented in Table 3.

$X_{1}$ (\% oil), $X_{2}$ (\% $S_{\text {mix }}$ ), $X_{3}$ (ultrasonication time; min), $X_{4}$ (ultrasonication intensity; \%), and $X_{5}$ (temperature; ${ }^{\circ} \mathrm{C}$ ) are the individual interactive effects which are shown in Table 3 as eqn (1). In eqn (1), \% oil and \% $S_{\text {mix }}$ individually show a positive effect, but a negative effect was also seen for the hydrodynamic diameter $\left(Y_{1}\right)$ with ultrasonication intensity followed by sonication time.

Correspondingly, a combination of independent variables showed a negative effect for (\% oil) $\times\left(\% S_{\text {mix }}\right)\left(X_{1} X_{2}\right)$ and $(\%$ $\left.S_{\text {mix }}\right) \times$ (sonication time) $\left(X_{2} X_{3}\right)$, and a positive effect for (\% oil) $\times\left(\%\right.$ ultrasonication intensity) $\left(X_{1} X_{4}\right)$ and (\% oil) $\times$ (temperature) $\left(X_{1} X_{5}\right)$, as shown in the 5-dimensional plot for particle size in Fig. 3A-E.

We observed that an increase in sonication time with concentration of $S_{\text {mix }}$ led to a reduction in globule size of NE. In 

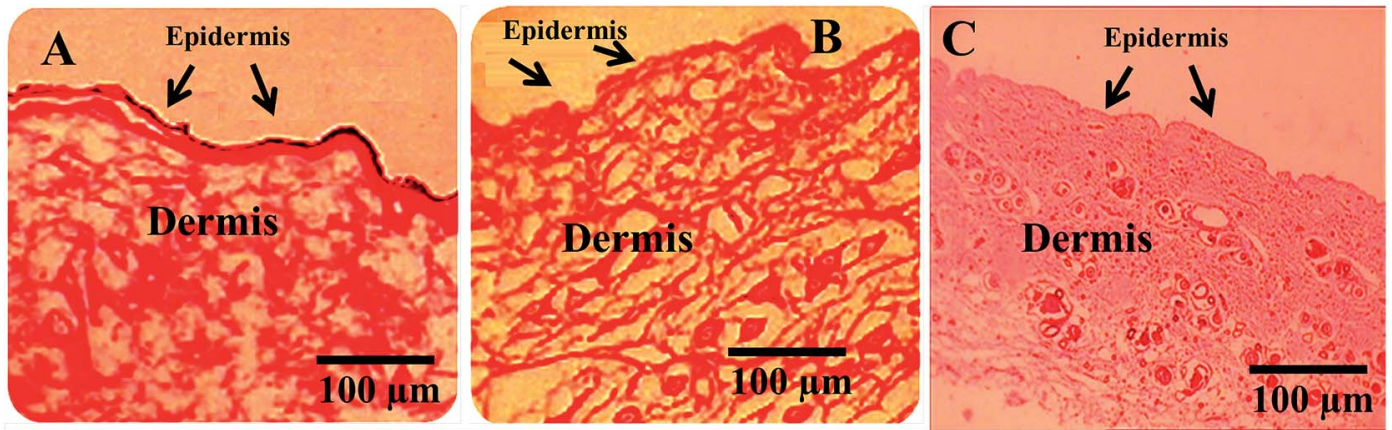

Fig. 10 Histopathology of skin: (A) control, (B) optimized Cur-NE treated rats; (C) fusidic acid treated rats.

this way, we saw a detrimental effect on the enhancement of oil concentration with the globule size of NE.

Likewise, we saw an increase in sonication time with a positive increase in $\%$ transmittance and $\% S_{\text {mix }}$, followed by an increase in \% oil in NE, as presented in eqn (2) and Fig. 3E-I. Moreover, a positive effect on $\%$ transmittance was shown due to the combined effect of $(\%$ oil $) \times\left(\% S_{\text {mix }}\right)$ $\left(X_{1} X_{2}\right),(\%$ oil $) \times\left(\%\right.$ ultrasonication intensity) $\left(X_{1} X_{4}\right)$, and $(\%$ $\left.S_{\text {mix }}\right) \times$ (temperature) $\left(X_{2} X_{5}\right)$. However, an insignificant effect was noted for $(\%$ oil $) \times($ temperature $)\left(X_{1} X_{5}\right),\left(\% S_{\text {mix }}\right)$ $\times\left(\%\right.$ ultrasonication intensity) $\left(X_{2} X_{4}\right)$, and (\% ultrasonication intensity) $\times$ (temperature) $\left(X_{2} X_{5}\right)$.

The effect on PDI from the independent variables is presented in eqn (3) and Fig. 3J-N, whereby an enhancement for PDI was found with an increase in sonication time and \% $S_{\text {mix }}$ counter to oil increase, which was not in supportive of $\%$ transmittance. As mixed independent variables, $(\%$ oil $) \times$ (ultrasonication time) $\left(X_{1} X_{4}\right),(\%$ oil $) \times($ temperature $)\left(X_{1} X_{5}\right)$, $\left(\% S_{\text {mix }}\right) \times$ (sonication time) $\left(X_{2} X_{3}\right),(\%$ ultrasonication intensity) $\times$ (temperature) $\left(X_{2} X_{5}\right)$, and (ultrasonication time) $\times$ (temperature) $\left(X_{3} X_{5}\right)$ exerted a negative magnitude, which proves a clear dominancy for ultrasonication time with $\%$ ultrasonication intensity on $\% S_{\text {mix }}$ and \% oil. This negative magnitude may be due to a reduction in globule size of NE; hence, it supports a lower PDI, due to ultrasonication time and ultrasonication intensity.

All the conditions were assessed for independent variables in order to find an optimized formulation (Table 5). The independent variables (\% oil and ultrasonication time) were put in a proper range while the constraint tried for $\%$ $S_{\text {mix }}$ was "reduce". On the other side, among dependent variables, "reduce" was labeled for globule size and "higher" was marked for PDI and \% transmittance.

On the basis of conditional parameters and quadratic equations, the five dependent variables were optimized for Cur-NE as forecast by CCD with final values of $5.00 \%$ oil, $10.0 \% S_{\text {mix }}, 10.0 \mathrm{~min}$ ultrasonication time, $40 \%$ ultrasonication intensity, and $50{ }^{\circ} \mathrm{C}$ temperature. Moreover, the globule size $(93.64 \pm 6.48 \mathrm{~nm}), \%$ transmittance $(98.64 \pm$ $0.37 \%)$, and PDI $(0.263 \pm 0.021)$ with demandable of 0.99 was predicted for the optimized Cur-NE.

\section{Optimized Cur-NE characterization}

A classic dye test was performed using methylene blue dye (water soluble) for optimized Cur-NE characterization. This test confirmed the oil in water nanoemulsion, with a maximum travel time as predicted. In addition, spreading of the dye was very fast.

\section{Nanoemulsion globule size, PDI and \% transmittance}

A particle size of $95.86 \mathrm{~nm}$ was predicted by "central composite design" whereas the observed particle size was $93.64 \pm 6.48 \mathrm{~nm}$ (Fig. 4A) along with the predicted value of 0.276 . The observed PDI value was $0.263 \pm 0.021$, suggesting a monomodal droplet size distribution (Fig. 4A). The "central composite design" software also gave a value of $99.01 \%$ for $\%$ transmittance, whereas the observed \% transmittance was $98.64 \pm 0.37 \%$ (Table 4).

\section{Zeta potential}

The graph in Fig. 4B shows a zeta potential of $-11.67 \pm 0.11 \mathrm{mV}$ for optimized Cur-NE. According to previously reported studies, the non-ionic surfactant stabilized oil droplets allow for this magnitude of droplet charge. ${ }^{27}$

\section{SEM and TEM study of the surface morphology}

SEM and TEM were used to determine shape and surface texture of Cur-NE. SEM showed the smooth and round surface of CurNE (Fig. 4C and D). TEM displayed the spherical particle shape of $<100 \mathrm{~nm}$ diameter, i.e. $93.64 \pm 6.48 \mathrm{~nm}$ globule size (Fig. 4D).

\section{Drug content, viscosity, refractive index (RI), and pH of Cur- NE}

Cur-NE showed fewer dense and clear formulations with an RI value of $1.51 \pm 0.043$. However, the viscosity of was $37 \pm 7 \mathrm{cp}$ and the $\mathrm{pH}$ was $7.4 \pm 0.07$. The drug content (Cur) was $98.11 \pm$ $0.16 \%$ for optimized Cur-NE (Table 5 ).

The drug content of the nanoemulsion formulation was measured by dissolving $1 \mathrm{ml}$ of the formulation in $10 \mathrm{ml}$ of methanol. This formulation was then placed in a shaking incubator (LSI-2005 RL, Lab Tech Co., Korea) (50 rpm at $37 \pm 0.5$ 
${ }^{\circ} \mathrm{C}$ ) for $30 \mathrm{~min}^{49}$ After $30 \mathrm{~min}$, the supernatant was collected and analyzed using a UHPLC-MS/MS method as previously reported by Ahmad et al. ${ }^{\mathbf{5 0}}$

\section{Differential scanning calorimetry (DSC)}

In agreement with the Certificate of Analysis (COA), the procured curcumin melting point range was observed to be 184-190 ${ }^{\circ} \mathrm{C}$. Cur showed a sharp endothermic peak at $186.0^{\circ} \mathrm{C}$ during DSC analysis (Fig. 5), confirming the crystalline structure of curcumin. DSC was also performed on Tween-80 (115.8 $\left.{ }^{\circ} \mathrm{C}\right)$, PEG-400 $\left(60.10{ }^{\circ} \mathrm{C}\right)$, and clove oil $\left(92.4{ }^{\circ} \mathrm{C}, 149.3{ }^{\circ} \mathrm{C}, 260.3\right.$ $\left.{ }^{\circ} \mathrm{C}\right)$. Cur-loaded-NE showed a two very small peaks for PEG-400 and Tween-80 in the thermogram of DSC. There was no peak for curcumin in the Cur-loaded-NE DSC thermogram. This means that Cur was completely entrapped inside the core of NE.

\section{In vitro drug release studies}

In the Fig. 6A, Cur-S was $73.23 \%$ in vitro released at $30 \mathrm{~min}$ followed by $100 \%$ release at $2.0 \mathrm{~h}$. On the other side, the in vitro release profile (Fig. 6A) of Cur from optimized Cur-NE was found to be biphasic with an initial burst release $(25.64 \pm 1.83 \%$ in $1 \mathrm{~h}$ ) followed by sustained release. Finally, 74.36\% Cur was released from Cur-NE, followed by up to $76.10 \%$. The initial burst release of Cur from Cur-NE might have occurred due to the erosion of superficial Cur in the Cur-NE. On the other side, the lipid that accommodated the drug inside the core during the formulation process of the nanoemulsion would be responsible for a sustained release profile. ${ }^{13,32}$ The release kinetics of Cur from optimized Cur-NE was fitted in various models and the in vitro drug release model showing highest value of $R^{2}$ was considered to be the best model for the release of Cur from Cur-NE. The highest value of the correlation coefficient $\left(R^{2}=0.9612\right)$ was observed for the Higuchi matrix, followed by the first-order $\left(R^{2}=0.9518\right)$, zero order $\left(R^{2}=0.9103\right)$, and Korsmeyer-Peppas. This release profile could be important, with an initial fast drug release to achieve the high concentration gradient required for successful transdermal drug delivery. ${ }^{13,32}$

\section{In vitro skin permeation study}

Rat skin was isolated for Cur-pure suspension from optimized Cur-NE to perform permeation studies (Fig. 6B). In the cumulative drug permeation study, the permeation of the Cur suspension was $20.36 \pm 3.78 \%$, while that for optimized Cur-NE was $79.64 \pm 5.34 \%$ after 24.0 h. In the optimized Cur-NE, Tween-80 is a polysorbate surfactant by nature; therefore, it possesses the capability to enhance drug permeation by penetrating into the intracellular regions of the stratum corneum and eventually solubilizing the lipid components. ${ }^{33,34}$

\section{Pharmacodynamic study}

\section{Wound healing evaluation}

Photographs of the wound healing effects of the control (placebo), optimized nanoemulsion without Cur, pure clove oil, pure Cur-S, optimized nanoemulsion (Cur-NE) and marketed preparation of antibiotic fusidic acid (Fusidin; positive control) treatments on wound area contraction of the rats are presented in Fig. 7. However, the results of the influence of pure clove oil, pure Cur-S, optimized Cur-NE and fusidic acid on wound area contraction of rats are presented in Fig. 8. These results show that wound contraction in the rats increased until day 24 in all three test samples (pure clove oil; pure Cur-S; and optimized Cur-NE) and fusidic acid treated rats. Pure clove oil and pure Cur-S significantly helped the wound contraction from day 12 to 24 when compared to control group $(P<0.05)$. Cur-NE and fusidic acid significantly helped in the wound contraction from day 12 to 24 when compared to the control group $(P<$ 0.01) in Fig. 8. Cur-NE showed the same effects on the treatment of wounds in comparison to fusidic acid, due to its nano-sized particles and penetration enhancement, leading to a more sustained released action. The results of the effects of the treatments (pure clove oil, pure Cur-S and Cur-NE) as compared to antibiotic fusidic acid on the epithelization period are shown in Fig. 9. The complete epithelization times were $15 \pm 1,13 \pm 1,12.5 \pm 1,9.5 \pm 0.7$ and $9.5 \pm 0.7$ days for control (placebo), pure clove oil, pure Cur-S and Cur-NE and antibiotic fusidic acid, respectively. The epithelization period was sufficiently shorter in Cur-NE and fusidic acid treated rats as compared to those treated with the control, pure clove oil, and pure Cur-S $(P<0.05)$. Wound contraction effects and epithelization in the Cur-NEtreated rats were highly comparable with those treated with antibiotic fusidic acid. It was observed from these results that pure clove oil and pure Cur-S were much more efficacious in a nanoemulsion in comparison with their pure forms. These results suggest that the developed nanoemulsion shows greater wound healing effects after topical application as compared to pure clove oil and pure Cur-S. Enhanced wound contraction by Cur-NE was possible due to the submicron-range droplets of the nanoemulsion and the presence of solubilizers such as Tween-80 and PEG in Cur-NE. The production of wound contraction using pure clove oil, pure Cur-S, and Cur-NE indicates that pure clove oil and pure Cur-S possess a definite healing action, as 100\% of wound healing was observed to be due to contraction. ${ }^{7-11}$ This wound healing potential of pure clove oil, pure Cur-S, and Cur-NE is probably due to definite enhancement in the proliferation of epithelial cells. ${ }^{10}$ The most important outcome of the current research is that pure clove oil, pure Cur-S and Cur-NE applied topically accelerated wound healing effects at a faster rate than the control in the developed rat excision wound model.

\section{Wound healing tissue evaluation by histopathology}

Histopathological wound healing tissue evaluation images for the control (without drug), test formulation (Cur-NE), and reference (fusidic acid treated) are presented in Fig. 10. Cur-NEtreated rats revealed a large amount of granulation tissue and a small amount of mononuclear inflammatory cells. We also found that there is no restoration of adnexa or extensive 
fibrosis, nor are there any signs of ulceration and edema (Fig. 10B).

The fusidic acid treated rats revealed well-formed healed skin structures, much closer to normal epidermis, and restoration of adnexa. We also found wide fibrosis and collagen tissue within the dermis (Fig. 10C).

\section{Anti-inflammatory activity}

Inflammation was assessed using a rat hind paw edema model for the current research study. ${ }^{\mathbf{1 3 , 4 5}}$ The inflammagen carrageenan was injected to develop the edema. Cur-NE exhibited significant reduction $(p<0.05)$ in inflammation $(48.23 \%)$ in comparison with pure Cur-S $(14.25 \%)$ in the rats. Cur-NE showed better results in the treatment of inflammation in the rat's paw significantly.

\section{Conclusion}

Cur-NE was prepared using an ultrasonication technique, chemical engineering, and "Quality by Design" (QbD) software. The highest NE region was shown in $S_{\text {mix }}$ (4:1 ratio) from different $S_{\text {mix }}$ pseudo-ternary phase diagrams. A fine NE from a coarse emulsion was prepared using ultrasonication techniques. SEM confirmed the smooth surface of NE, while the globular size of Cur-NE was further confirmed by TEM. Further characterization of Cur-NE, including confirming the entrapment of Cur inside the core of NE, was performed using DSC, whereas the maximum permeation of curcumin from Cur-NE was revealed in the ex vivo skin permeation studies. Wound healing effects, potentially enhanced by Cur-NE, were statistically significant, as compared to pure clove oil, Cur-S and the control. Cur-NE wound healing effects were shown to be significantly similar to those promoted by the antibiotic fusidic acid. No signs of inflammatory cells were seen in the histopathological examination of wounds treated by Cur-NE. These results prove that optimized Cur-NE is safe and nontoxic. All the results are based on the topical delivery of curcumin from optimized Cur-NE followed by the enhancement of wound healing effects in rats. In parallel, optimized Cur-NE exhibited highly effective anti-inflammatory activity in the healing of edema compared with Cur-S in the carrageenan-induced ratpaw edema model.

\section{Conflicts of interest}

No conflict of interests exists among authors. No grants were received.

\section{Acknowledgements}

The authors are very thankful to Prof. (Dr) Mastour Safar AlGhamdi who helped in the wounds healing evaluations. All animal procedures were performed in accordance with the Guidelines for Care and Use of Laboratory Animals of "Imam Abdulrahman Bin Faisal University" and approved by the "Institutional Animal Care and Use Committee (IACUC)" with
"Institutional Review Board (IRB Approval Number: IRB-UGS2018-05-200)". Humans were NOT used in this research study, only animals (rats) were used.

\section{References}

1 M. Singh, R. Govindarajan, V. Nath, A. K. S. Rawat and S. Mehrotra, Antimicrobial, Wound Healing and Antioxidant Activity of Plagiochasma Appendiculatum Lehm. et Lind, J. Ethnopharmacol., 2006, 107(1), 67-72.

2 I. B. Pathan, S. J. Munde, S. Shelke, W. Ambekar and C. M. Setty, Curcumin loaded fish scale collagen-HPMC nanogel for wound healing application: ex vivo and in vivo evaluation, Int. J. Polym. Mater., 2019, 68(4), 165-174.

3 L. Gould, P. Abadir, H. Brem, et al., Chronic wound repair and healing in older adults: current status and future research, J. Am. Geriatr. Soc., 2015, 63(3), 427-438.

$4 \mathrm{~J}$. Boateng and O. Catanzano, Advanced therapeutic dressing for effective wound healing-review, J. Pharm. Sci., 2015, 104(11), 3653-3680.

5 A. Stejskalova and B. D. Almquist, Using biomaterials to rewire the process of wound repair, Biomater. Sci., 2017, 5(8), 1421-1434.

6 F. Saporito, G. Sandri, M. C. Bonferoni, S. Rossi, C. Boselli, A. Icaro Cornaglia, B. Mannucci, P. Grisoli, B. Vigani and F. Ferrari, Essential oil-loaded lipid nanoparticles for wound healing, Int. J. Nanomed., 2017, 13, 175-186.

7 S. D. Fitzmaurice, R. K. Sivamani and R. R. Isseroff, Antioxidant therapies for wound healing: a clinical guide to currently commercially available products, Skin Pharmacol. Physiol., 2011, 24(3), 113-126.

8 L. Thomas, F. Zakir, M. A. Mirza, M. K. Anwer, F. J. Ahmad and Z. Iqbal, Development of Curcumin loaded chitosan polymer based nanoemulsion gel: in vitro, ex vivo evaluation and in vivo wound healing studies, Int. J. Biol. Macromol., 2017, 101, 569-579.

9 D. Gopinath, M. R. Ahmed, K. Gomathi, K. Chitra, P. K. Sehgal and R. Jayakumar, Dermal wound healing processes with curcumin incorporated collagen films, Biomaterials, 2004, 25, 1911-1917.

10 G. S. Sidhu, A. K. Singh, D. Thaloor, K. K. Banaudha, G. K. Patnaik, R. C. Srimal and R. K. Maheshwari, Enhancement of wound healing by curcumin in animals, Wound Repair Regen., 1998, 6(2), 167-177.

11 C. Gong, Q. Wu, Y. Wang, D. Zhang, F. Luo, X. Zhao, Y. Wei and Z. Qian, A biodegradable hydrogel system containing curcumin encapsulated in micelles for cutaneous wound healing, Biomaterials, 2013, 34, 6377-6387.

12 A. Teichmann, S. Heuschkel, U. Jacobi, G. Presse, R. H. Neubert, W. Sterry and J. Lademann, Comparison of stratum corneum penetration and localization of a lipophilic model drug applied in an o/w microemulsion and an amphiphilic cream, Eur. J. Pharm. Biopharm., 2007, 67(3), 699-706.

13 N. Ahmad, M. A. Alam, F. J. Ahmad, M. Sarafroz, K. Ansari, S. Sharma and M. Amir, Ultrasonication techniques used for the preparation of novel eugenol-nanoemulsion in the 
treatment of wounds healings and anti-inflammatory, $J$. Drug Delivery Sci. Technol., 2018, 46, 461-473.

14 S. Wang, F. Zheng, Y. Huang, Y. Fang, M. Shen, M. Zhu and X. Shi, Encapsulation of amoxicillin within laponite-Doped poly(lactic-co-glycolic acid) nanofibers: preparation characterization, and antibacterial activity, ACS Appl. Mater. Interfaces, 2012, 4, 6393-6401.

15 M. K. Anwer, S. Jamil, E. O. Ibnouf and F. Shakeel, Enhanced antibacterial effects of clove essential oil by nanoemulsion, $J$. Oleo Sci., 2014, 63, 347-354.

16 E. Abd, S. Namjoshi, Y. H. Mohammed, M. S. Roberts and J. E. Grice, Synergistic skin penetration enhancer and nanoemulsion formulations promote the human epidermal permeation of caffeine and naproxen, J. Pharm. Sci., 2016, 105, 212-220.

17 H. P. Thakkar, A. Khunt, R. D. Dhande and A. A. Patel, Formulation and evaluation of Itraconazole nanoemulsion for enhanced oral bioavailability, J. Microencapsulation, 2015, 32, 559-569.

18 B. Baroli, M. A. López-Quintela, M. B. Delgado-Charro, A. M. Fadda and J. Blanco-Méndez, Microemulsions for topical delivery of 8-methoxsalen, J. Controlled Release, 2000, 69(1), 209-218.

19 T. Tadros, P. Izquierdo, J. Esquena and C. Solans, Formation and stability of nano-emulsions, Adv. Colloid Interface Sci., 2004, 108-109, 303-318.

20 D. J. McClements, Nanoemulsions versus microemulsions: terminology, differences, and similarities, Soft Matter, 2012a, 8, 1719-1729.

21 M. Sivakumar, S. Y. Tang and K. W. Tan, Cavitation technology - a greener processing technique for the generation of pharmaceutical nanoemulsions, Ultrason. Sonochem., 2014, 21(6), 2069-2083.

22 I. Suntar, E. K. Akkol, A. Tosun and H. Keles, Comparative pharmacological and phytochemical investigation on the wound-healing effects of the frequently used essential oils, J. Essent. Oil Res., 2014, 26, 41-49.

23 R. Parveen, S. Baboota, J. Ali, A. Ahuja, S. S. Vasudev and S. Ahmad, Oil based nanocarrier for improved oral delivery of silymarin: in vitro and in vivo studies, Int. J. Pharm., 2011, 413, 245-253.

24 Po-H. Li and B.-H. Chiang, Process optimization and stability of D-limonenein-water nanoemulsions prepared by ultrasonic emulsification using response surface methodology, Ultrason. Sonochem., 2012, 19, 192-197.

25 S. K. Singh, P. Dadhania, P. R. Vuddanda, A. Jain, S. Velaga and S. Singh, Intranasal delivery of asenapine loaded nanostructured lipid carriers: formulation, characterization, pharmacokinetic and behavioural assessment, RSC Adv., 2016, 6, 2032-2045.

26 T. Delmas, H. Piraux, A. C. Couffin, I. Texier, F. Vinet, P. Poulin, M. E. Cates and J. Bibette, How to prepare and stabilize very small nanoemulsions, Langmuir, 2011, 27(5), 1683-1692.

27 V. Ghosh, A. Mukherjee and N. Chandrasekaran, Ultrasonic emulsification of food-grade nanoemulsion formulation and evaluation of its bactericidal activity, Ultrason. Sonochem., 2013, 20(1), 338-344.

28 P. H. Li and B. H. Chiang, Process optimization and stability of D-limonene-in-water nanoemulsions prepared by ultrasonic emulsification using response surface methodology, Ultrason. Sonochem., 2012, 19(1), 192-197.

29 N. C. Loong, M. Basri, L. F. Fang, H. R. F. Masoumi, M. Tripathy, R. A. Karjiban and E. Abdul-Malek, Comparison of Box-Behnken and central composite designs in optimization of fullerene loaded palm-based nano-emulsions for cosmeceutical application, Ind. Crops Prod., 2014, 59, 309-317.

30 E. Gonzalez-Mira, M. A. Egea, E. B. Souto, A. C. Calpena and M. L. García, Optimizing flurbiprofen-loaded NLC by central composite factorial design for ocular delivery, Nanotechnology, 2011, 22(4), 045101.

31 S. F. Tan, H. R. Masoumi, R. A. Karjiban, J. Stanslas, B. P. Kirby, M. Basri and H. B. Basri, Ultrasonic emulsification of parenteral valproic acid-loaded nanoemulsion with response surface methodology and evaluation of its stability, Ultrason. Sonochem., 2016, 29, 299-308.

32 N. Ahmad, R. Ahmad, M. A. Alam, F. J. Ahmad and M. Amir, Impact of ultrasonication techniques on the preparation of novel Amiloride-nanoemulsion used for intranasal delivery in the treatment of epilepsy, Artif. Cells, Nanomed., Biotechnol., 2018, 46(suppl. 3), S192-S207.

33 N. Ahmad, M. A. Alam, F. J. Ahmad, M. Sarafroz, K. Ansari, S. Sharma and M. Amir, Ultrasonication techniques used for the preparation of novel eugenol-nanoemulsion in the treatment of wounds healings and anti-inflammatory, $J$. Drug Delivery Sci. Technol., 2018, 46, 461-473.

34 N. Ahmad, R. Ahmad, A. A. Naqvi, M. A. Alam, M. Ashafaq, R. Abdur Rub and F. J. Ahmad, Intranasal delivery of quercetin-loaded mucoadhesive nanoemulsion for treatment of cerebral ischaemia, Artif. Cells, Nanomed., Biotechnol., 2018, 46(4), 717-729.

35 S. Sood, K. Jain and K. Gowthamarajan, Optimization of curcumin nanoemulsion for intranasal delivery using design of experiment and its toxicity assessment, Colloids Surf., B, 2014, 113, 330-337.

36 N. Ahmad, R. Ahmad, A. A. Naqvi, M. A. Alam, M. Ashafaq, Z. Iqbal and F. J. Ahmad, Isolation, characterization, and quantification of curcuminoids and their comparative effects in cerebral ischemia, J. Liq. Chromatogr. Relat. Technol., 2017, 40(3), 133-146.

37 S. Ahmed, A. Gull, M. Alam, M. Aqil and Y. Sultana, Ultrasonically tailored, chemically engineered and "QbD" enabled fabrication of agomelatine nanoemulsion; optimization, characterization, ex vivo permeation and stability study, Ultrason. Sonochem., 2018, 41, 213-226.

38 F. Shakeel, P. Alam, M. K. Anwer, S. A. Alanazi, I. A. Alsarra and M. H. Alqarni, Wound healing evaluation of selfnanoemulsifying drug delivery system containing Piper cubeba essential oil, 3 Biotech, 2019, 9(3), 82.

39 A. A. Moghaddam, A. Ahad, M. Aqil, F. J. Ahmad, Y. Sultana and A. Ali, Ibuprofen loaded nano-ethanolic liposomes 
carbopol gel system: in vitro characterization and antiinflammatory efficacy assessment in Wistar rats, J. Polym. Eng., 2018, 38(3), 291-298.

40 V. Ghosh, A. Mukherjee and N. Chandrasekaran, Ultrasonic emulsification of food-grade nanoemulsion formulation and evaluation of its bactericidal activity, Ultrason. Sonochem., 2013, 20(1), 338-344.

41 P. Alam, M. J. Ansari, M. K. Anwer, M. Raish, Y. K. Kamal and F. Shakeel, Wound healing effects of nanoemulsion containing clove essential oil, Artif. Cells, Nanomed., Biotechnol., 2017, 45(3), 591-597.

42 N. Ahmad, R. Ahmad, M. A. Alam and F. J. Ahmad, Quantification and Brain Targeting of Eugenol-Loaded Surface Modified Nanoparticles Through Intranasal Route in the Treatment of Cerebral Ischemia, Drug Res., 2018, 68(10), 584-595.

43 S. V. Kothiwale, V. Patwardhan, M. Gandhi, R. Sohoni and A. Kumar, A comparative study of antiplaque and antigingivitis effects of herbal mouthrinse containing tea tree oil, clove, and basil with commercially available essential oil mouthrinse, J. Indian Soc. Periodontol., 2014, 18(3), 316-320.

44 D. J. McClements and H. Xiao, Potential biological fate of ingested nanoemulsions: influence of particle characteristics, Food Funct., 2012, 3(3), 202-220.

45 C. A. Winter, E. A. Risley and G. W. Nuss, Anti-Inflammatory and Antipyretic Activities Of Indomethacin, 1-(p-
Chlorobenzoyl)-5-Methoxy-2-Methylindole-3-Acetic Acid, J. Pharmacol. Exp. Ther., 1963, 141, 369-376.

46 R. Ahmad, A. A. Naqvi, N. Ahmad, M. Baraka, M. Mastour, S. Al Sharedah, et al., Awareness, percepton, attitude, and knowledge regarding complementary and alternative medicines (CAMs) among the pharmacy and medical students public university in Saudi Arabia, Arch. Pharm. Pract., 2017, 8, 51-63.

47 R. Ahmad, A. A. Naqvi, H. M. Al-Bukhaytan, A. H. Al-Nasser and A. H. Baqer Al-Ebrahim, Evaluation of aromatherapy with lavender oil on academic stress: a randomized placebo controlled clinical trial, Contemp. Clin. Trials Commun., 2019, 14, 100346.

48 https://upnature.com/blogs/news/top-16-best-essential-oilsfor-energy-and-5-amazing-blends.

49 M. Laxmi, A. Bhardwaj, S. Mehta and A. Mehta, Development and characterization of nanoemulsion as carrier for the enhancement of bioavailability of artemether, Artif. Cells, Nanomed., Biotechnol., 2015, 43(5), 334-344.

50 N. Ahmad, I. Ahmad, S. Umar, Z. Iqbal, M. Samim and F. J. Ahmad, PNIPAM nanoparticles for targeted and enhanced nose-to-brain delivery of curcuminoids: UPLC/ ESI-Q-ToF-MS/MS-based pharmacokinetics and pharmacodynamic evaluation in cerebral ischemia model, Drug Delivery, 2016, 23(7), 2095-2114. 